\title{
Extensive intraoperative peritoneal lavage and chemotherapy for gastric cancer patients with peritoneal free cancer cells
}

\author{
Shinya Shimada ${ }^{1,2}$, Eiji Tanaka ${ }^{2}$, Takashi Marutsuka ${ }^{2}$, Ubehiko Honmyo ${ }^{2}$, Hidehiro Tokunaga ${ }^{3}$, \\ Yasushi YAGI $^{4}$, Norimitsu AOKI ${ }^{1}$, and Michio OgaWA ${ }^{2}$ \\ ${ }^{1}$ Department of Surgery, Yatsushiro Health Insurance General Hospital, Kumamoto 866-8660, Japan \\ ${ }^{2}$ Second Department of Surgery, Kumamoto University School of Medicine, Kumamoto, Japan \\ ${ }^{3}$ Department of Surgical Pathology, Kumamoto University School of Medicine, Kumamoto, Japan \\ ${ }^{4}$ Department of Surgery, Kumamoto Regional Hospital, Kumamoto, Japan
}

\begin{abstract}
The effects of extensive intraoperative peritoneal lavage (EIPL) for gastric cancer patients with peritoneal free cancer cells were investigated. This study was based on 22 consecutive patients with peritoneal free cancer cells, among 663 patients who underwent curative surgical treatment for advanced gastric cancer. The 22 patients were followed up for 2 years or until death. These patients were divided into three groups: group 1, patients with no additional intraoperative therapy (from 1989 to $1992 ; n=8$ ); group 2, patients with intraoperative intraperitoneal chemotherapy alone (from 1992 to 1995; $n=7$ ); and group 3, patients with EIPL followed by intraoperative intraperitoneal chemotherapy (from 1996 to 1999; $n=7)$. Reverse transcriptase-polymerase chain reaction (RT-PCR) analysis showed that viable cancer cells were not detected after the eighth EIPL in a gastric cancer patient with numerous intraperitoneal free cancer cells. In group 3, 4 of the 7 patients survived for more than 2 years, including 3 with cancer-free status, whereas no patient survived cancer-free in groups 1 and 2. The peritoneal recurrence rates and cancerspecific 2-year survival rates in groups 1, 2, and 3 were $100 \%$, $85.7 \%$ and $42.9 \%$; and $0 \%, 14.3 \%$, and $57.1 \%$, respectively. The 2-year survival rate of group 3 was significantly higher than that of group $1(P=0.017)$ and that of group $2(P=$ $0.025)$. In a subset analysis, patients with peritoneal free gastric cancer cells but no macroscopic dissemination showed a statistically significant improvement in survival those treated with EIPL compared with those not treated with EIPL.
\end{abstract}

Key words Gastric carcinoma - Peritoneal free cancer cells · Dissemination $\cdot$ Peritoneal lavage $\cdot$ Chemotherapy

Offprint requests to: S. Shimada

Received: June 10, 2002 / Accepted: June 25, 2002
Introduction

Peritoneal metastasis is the most frequent cause of death in patients with advanced gastric cancer [1-3]. In patients with serosal invasion, about half of the patients develop peritoneal recurrence and die of this disease during the first 2 years of follow-up, even if curative resection is performed [4]. It has been reported that the 5-year survival rate of patients with positive lavage cytology is only $2 \%$ [5]. Although the prognosis of patients with macroscopic dissemination of gastric carcinoma into the peritoneal cavity has proven to be disappointing [6], adjuvant intraperitoneal therapeutic regimens with curative intent might be promising in patients with intraperitoneal free cancer cells without macroscopic peritoneal carcinomatosis. This is because the presence of intraperitoneal free cancer cells without macroscopic dissemination could possibly mean a condition wherein the implantation of cancer cells on the intraperitoneal wall has not yet occurred. If this supposition is true, the number of intraperitoneal free cancer cells could be reduced to almost zero using the method of so-called "limited dilution". If 1 liter of saline lavage reduces the number of peritoneal free cancer cells to at least one-tenth, ten such washings should lead to a logarithmic reduction of cancer cells of approximately $1: 10^{10}$. However, because the peritoneal cavity is not a simple container, this procedure might not work as well as could be expected. If this were the case, intraperitoneal chemotherapy could be effective for eradicating the remaining cancer cells. In addition, if there were only a few cancer cells, these cells cells might find it hard to survive and form macroscopic dissemination, because the chance of implantation would be markedly reduced by the small number of viable cancer cells [7].

Therefore, we attempted extensive lavage after curative resection prior to intraperitoneal chemotherapy for gastric cancer patients with peritoneal free cancer cells, and evaluated the clinical effects. 


\section{Patients}

Between 1989 and 1999, 663 patients with advanced gastric carcinoma underwent potentially curative operation with extended lymph node dissection at the Department of Surgery, Yatsushiro Health Insurance General Hospital, and the Second Department of Surgery, Kumamoto University Medical School, and a total of 22 consecutive patients who had peritoneal free cancer cells by cytological examination but had no macroscopic dissemination was selected. These patients were divided into three groups: group 1, patients without extensive intraoperative peritoneal lavage (EIPL) or chemotherapy (from 1989 to $1992 ; n=8$ ); group 2, patients with only intraoperative intraperitoneal chemotherapy (from 1993 to 1995; $n=7$ ); and group 3, patients with both the EIPL and chemotherapy (from 1996 to 1999; $n=7$ ). Pathologic diagnosis and classifications were based on the Japanese classification of gastric carcinoma outlined by the Japanese Research Society for Gastric Cancer [8]. All patients received adjuvant chemotherapy (oral administration of 5fluorouracil derivatives) after the surgical treatment, and all gave their informed consent.

\section{Cytological detection of free cancer cells in the peritoneal cavity}

The surgical tumor stage was sufficiently examined after laparotomy. When no macroscopic dissemination was detected, $100 \mathrm{ml}$ of physiologic saline was introduced into the abdominal cavity and manually dispersed. The introduced fluid was collected from several regions of the abdominal cavity, such as the left and right subphrenic areas and the pouch of Douglas. Immediately after the collection, $1 \mathrm{ml}$ of heparin was added to the fluid. Half of the fluid was centrifuged at $1000 \mathrm{~g}$ for $10 \mathrm{~min}$, and the nucleated cell layer was smeared onto a glass slide and stained according to the Papanicolaou, Giemsa, and periodic acid Schiff (PAS) methods. The remaining fluid was used for reverse transcriptasepolymerase chain reaction (RT-PCR) analysis.

\section{Extensive intraoperative peritoneal lavage (EIPL) and chemotherapy}

Immediately after the cytological report showing positivity for peritoneal malignant cells, the contents of the peritoneal cavity were diluted with 1 liter of physiologic saline and completely aspirated, this procedure being done three times. After the potentially curative operation was performed, the peritoneal cavity was extensively washed, using 7 liters of physiologic saline (1 liter, seven times) followed by intraperitoneal chemotherapy, and $200 \mathrm{ml}$ of each sample was collected for consecutive cytological and RT-PCR analysis. After the extensive washing and aspiration, $100 \mathrm{mg}$ of cisplatin (CDDP) was administered into the peritoneal cavity. Peritoneal lavage for groups 1 and 2 was done with 3 liters (1 liter, three times) before closure of the abdominal wall or chemotherapy, respectively.

\section{RT-PCR}

The lavage samples (approximately $200 \mathrm{ml}$ ) were centrifuged at $1000 \mathrm{~g}$ for $10 \mathrm{~min}$ at $4^{\circ} \mathrm{C}$ to collect intact cells, and resuspended in $1 \mathrm{ml}$ of TRIzol Reagent (Invitrogen, Carlsbad, CA, USA). Total RNAs were isolated according to the guanidinium-thiocyanate-phenol-chloroform method [9]. The cDNA was synthesized from approximately $1 \mu \mathrm{g}$ of the total RNA. The presence of carcinoembryonic antigen (CEA) cDNA in the reverse transcription products was detected by PCR amplification in separate reactions, using oligonucleotide primers of this gene. PCR was carried out as described by Gerhard et al. [10]. To ensure that the RNA was of sufficient purity for RT-PCR, a PCR assay with primers specific for a housekeeping gene, glyceraldehyde-3phosphate dehydrogenase (GAPDH) cDNA was carried out in each sample, except that only 35 cycles were performed, under the following cycling conditions: $1 \mathrm{~min}$ at $94^{\circ} \mathrm{C}, 2 \mathrm{~min}$ at $56^{\circ} \mathrm{C}$, and $2 \mathrm{~min}$ at $72^{\circ} \mathrm{C}$. The primer sequences for GAPDH were: $5^{\prime}$-ATGGA TGATGATATCGCCGCG-3' and 5'-CTAGAAGCA TTTGCGGTGGACGATGGAGG GGCC-3'. Firststrand cDNA was synthesized using SuperScript II reverse transcriptase (Gibco BRL, Gaithersburg, $\mathrm{MD}$, USA) and random hexanucleotide primers (Pharmacia Biotech, Uppsala, Sweden) according to the manufacturer's instructions. CEA-specific oligonucleotide primers synthesized for the nested RTPCR analyses were those reported by Gerhard et al. [10]. The first PCR amplification was performed using primers CEA-A and CEA-B, followed by a second PCR amplification using primers CEA-C and CEA-B. The CEA primer sequences were: CEA-A, 5'TCTGGAAC TTCTCCTGGTCTCTCAGCTGG-3'; CEA-B, 5'-TGTAGCTGTTGCAAATGCTTTAAGG AAGAAGC-3'; and CEA-C, 5'-GGGCCACTGTCG GCATCA TGATTGG-3'. For the first PCR amplification, $1 \mu \mathrm{l}$ of the cDNA synthesis mix was used in a final volume of $25 \mu \mathrm{l}$. The amplification conditions included denaturation at $95^{\circ} \mathrm{C}$ for $30 \mathrm{~s}$, annealing at $63^{\circ} \mathrm{C}$ for $1 \mathrm{~min}$, and extension at $72^{\circ} \mathrm{C}$ for $1 \mathrm{~min}$, for 30 cycles, followed by a final extension step at $72^{\circ} \mathrm{C}$ for $5 \mathrm{~min}$. The second nested PCR amplification was performed in the same way, using $2 \mu \mathrm{l}$ of the first PCR reaction mix and the primers CEA-C and CEA-B. The amplification con- 
ditions were the same as those for the first PCR, except for the annealing, which was done at $60^{\circ} \mathrm{C}$. The external (first) CEA RT-PCR resulted in a 160-bp fragment, and the internal (second) PCR amplification yielded a 131bp product.

\section{RT-PCR analysis of fluid obtained from the EIPL}

We were very interested in determining when the malignant cells disappeared from the lavage fluid, at the level of molecular biological detection, as a result of the frequent and extensive washing, aspiration, and dilution. Therefore, the RT-PCR method was applied to each batch of lavage fluid in one patient in group 3 who had numerous free cancer cells in the peritoneal cavity at the time of laparotomy, according to the result of cytological examination (Fig. 1). Cytological examination revealed that cancer cells were not detected in the washing fluid after the fourth lavage, except for a detection in the washing fluid after the sixth lavage, in which only one cluster of cancer cells was detected. In the RT-PCR analysis using specific primers for CEA, no RT-PCR product was detected in the washing fluid after the eighth lavage. On the other hand, the RT-PCR product of CEA was observed in all the batches of lavage fluid of a patient with visible peritoneal dissemination. Based on these RT-PCR analyses, it was assumed that extensive washing using 10 liters of physiologic saline (1 liter, ten times) might flush out the free cancer cells from the peritoneal cavity according to the law of "limited dilution".

\section{Impact of EIPL on recurrence pattern and 2-year survival rate}

All patients who underwent potentially curative resection without EIPL or chemotherapy (group 1) had peritoneal recurrence $(100 \%)$ and died within 2 years. All but one of the patients who underwent curative resec- tion with intraperitoneal chemotherapy alone (group 2) also died within 2 years. However, the recurrence pattern in the group 2 patients included a patient who died of lung metastasis. One patient survived for more than 2 years with peritoneal recurrence. The overall peritoneal recurrence rate during 2-year follow-up was $85.7 \%$ (six of seven) in group 2. On the other hand, in the patients who underwent curative resection with both the EIPL and chemotherapy (group 3), only three of the seven patients $(42.9 \%)$ had peritoneal recurrence. The remaining four patients have not had peritoneal recurrence during 2-year follow-up and are still alive, although one of the four patients has skin recurrence. The 2-year survival rates of groups 1,2 , and 3 were 0 , $14.3 \%$ and $57.1 \%$, respectively. The Kaplan-Meier survival curves for the three studied groups are shown in Fig. 2. The cancer-specific 2-year survival curve of group 3 differed significantly from those of group 1 and group $2(P=0.017$ and $P=0.025$, respectively). There was no significant difference between group 1 and group 2 .

\section{Discussion}

Attention has been directed toward the detection of peritoneal free cancer cells to predict the prognosis of patients with advanced gastric carcinoma without overt peritoneal carcinomatosis [11]. However, the results of such studies have not been fully reflected in the treatment. On the other hand, some prospective randomized trials of early postoperative intraperitoneal chemotherapy as an adjuvant to resectable gastric cancer have shown that patients with stage III gastric cancer had a statistically significant improvement in survival when treated with perioperative chemotherapy [12]. Intraperitoneal chemotherapy has been designed to eradicate residual microscopic disease present in the peritoneal cavity after cancer resection. These studies would suggest that intraperitoneal chemotherapy could be effective to eradicate a few residual carcinoma cells. Because the small water-soluble molecules of commonly used

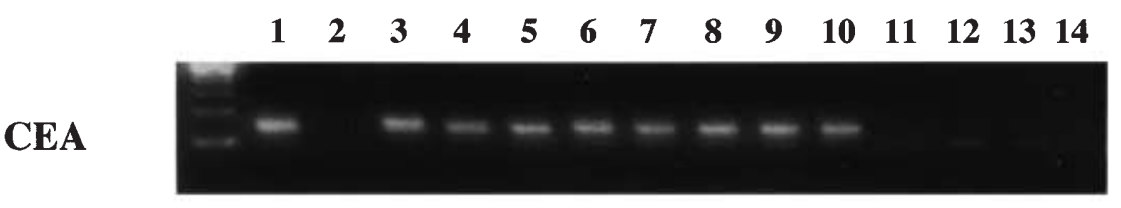

GAPDH

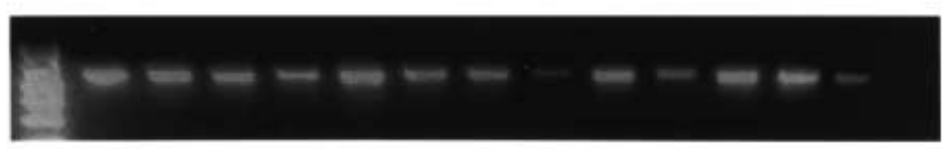

Cytology

$$
++++-+----
$$

Fig. 1. Reverse-transcriptase-polymerase chain reaction (RT-PCR) analysis using specific primers for carcinoembryonic antigen ( $C E A)$ and cytological examination of serial batches of fluid from extensive intraoperative peritoneal lavage. Lane 1, Total RNA from MCF7 cell; lane 2, total RNA from lavage fluid of a patient with early gastric carcinoma; lane 3, total RNA from the cytologypositive patient before extensive lavage; lanes 4-13, total RNA from lavages 110 , respectively; lane 14 , distilled water. $G A P D H$, Glyceraldehyde 3-phosphate dehydrogenase 


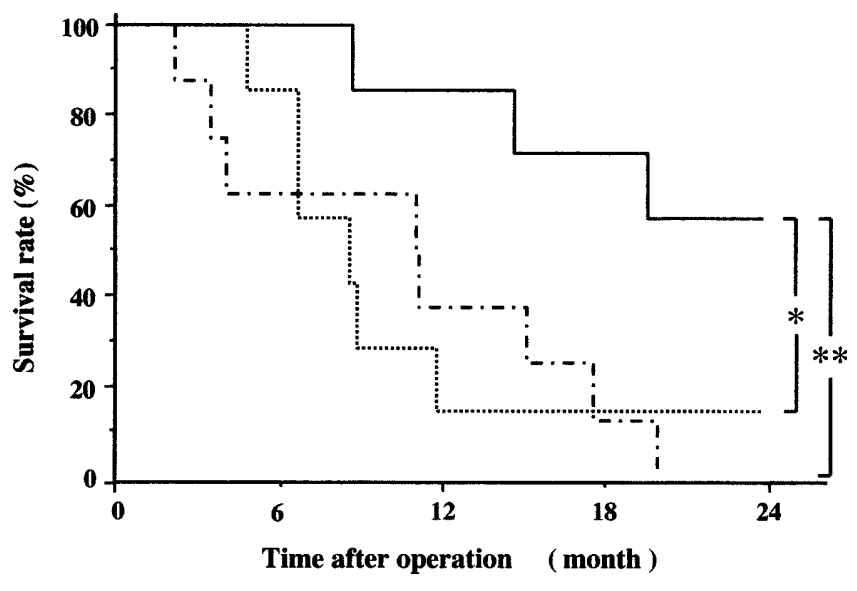

Fig. 2. Cancer-specific Kaplan-Meier survival curves for the three studied groups. Broken line, Patients who underwent potentially curative resection without extensive intraoperative peritoneal lavage or chemotherapy (group 1). Dotted line, Patients with intraperitoneal chemotherapy alone (group 2). Solid line, Patients with both the extensive intraoperative peritoneal lavage and chemotherapy (group 3 ). ${ }^{*} P=0.025$; $* * P=0.017$

anticancer drugs, such as CDDP or mitomycin $\mathrm{C}$, are rapidly absorbed through capillary walls in the subperitoneum, an effective concentration of the drugs is not maintained for long in the peritoneal cavity, resulting in such treatment playing an insufficient role to eradicate a large number of cancer cells [13]. The present study clearly showed that the peritoneal recurrence rate was substantially reduced when EIPL and chemotherapy were performed (group 3). Recently, it has been reported that the use of a peritoneal immunological method, with a combination of selected monoclonal antibodies, allowed the identification of cytologically false-negative cases in gastric cancer [11]. In these studies, Kaplan-Meier curves showed a statistically significant decrease of peritoneal recurrence in patients with peritoneal free cancer cells detected by immunocytochemical methods compared with the findings in patients with peritoneal free cancer cells detected morphologically. These data suggest that immunocytochemical detection is more sensitive than morphological cytology, and that gastric cancer patients with a smaller number of peritoneal free cancer cells than can be identified morphologically tend not to develop peritoneal recurrence. Sensitive methods, such as the immunological staining of cancer cells or RT-PCR examination for the detection of malignant cells, may be useful to select patients for the kind of treatment used in the present study (i.e., EIPL). However, because these methods are not generally available at the time of operation, cytological examination is commonly used to detect the free cancer cells in the peritoneal cavity. Therefore, at the present time, EIPL should be em- ployed for all gastric cancer patients with serosal invasion, including those with cytologically detected free cancer cells, because intraperitoneal free cancer cells have been detected by an immunological method in many patients with serosal invasion [11].

Free cancer cells in the peritoneal cavity are certainly important for the development of peritoneal recurrence in patients with gastric cancer. Frequent washing, and complete aspiration and dilution may not be an interesting procedure at the end of an operation, but it is quite a powerful method for reducing the number of cells to, potentially, zero, just like the so-called "limited dilution". Furthermore, because EIPL followed by intraperitoneal chemotherapy is simple, not so timeconsuming, and inexpensive, it can be performed anywhere and at any time. The use of EIPL may be indicated not only for patients with peritoneal gastric free cancer cells but also for those with gastric cancer invaded to the serosa. Thus, this protocol needs to be examined in larger scale trials and subjected to randomized controlled trials in the near future.

Acknowledgments This work was supported in part by Grants-in-Aid (no. 12877194 and no. 11671254) for Scientific Research from the Ministry of Education, Japan.

\section{References}

1. Balfour DC. Factors of significance in the prognosis of cancer of the stomach. Ann Surg 1973;105:733-40.

2. Maruyama K. The most important prognostic factors for gastric cancer patients. A study using univariate and multivariate analyses. Scand J Gastroenterol 1987;22 (Suppl 133):63-8.

3. Kaibara N, Iitsuka Y, Kimura A, Kobayashi Y, Hirooka Y, Nishidori $\mathrm{H}$, et al. Relationship between area of serosal invasion and prognosis in patients with gastric carcinoma. Cancer 1987;60: 136-9.

4. Ribeiro U Jr, Gama-Rodrigues JJ, Safatle-Ribeiro AV, Bitelman B, Ibrahim RE, Ferreira MB, et al. Prognostic significance of intraperitoneal free cancer cells obtained by laparoscopic peritoneal lavage in patients with gastric cancer. J Gastrointest Surg 1998;2:244-9.

5. Bando E, Yonemura Y, Takeshita Y, Taniguchi K, Yasui T, Yoshimitsu Y, et al. Intraoperative lavage for cytological examination in 1297 patients with gastric carcinoma. Am J Surg 1999; 178:256-62.

6. Sautner T, Hofbauer F, Depisch D, Schiessel R, Jakesz R. Adjuvant intraperitoneal cisplatin chemotherapy does not improve long-term survival after surgery for advanced gastric cancer. $\mathbf{J}$ Clin Oncol 1994;12:970-4.

7. Iitsuka Y, Kaneshima S, Tanida O, Takeuchi T, Koga S. Intraperitoneal free cancer cells and their viability in gastric cancer. Cancer 1979;44:1476-80.

8. Japanese Research Society for Gastric Cancer. Japanese classification of gastric carcinoma. 1st English Ed. Tokyo: Kanehara; 1999.

9. Chomczynski P, Sacchi N. Single-step method of RNA isolation by acid guanidinium thiocyanate-phenol-chloroform extraction. Anal Biochem 1987;162:156-9. 
10. Gerhard M, Juhl H, Kalthoff H, Schreiber HW, Wagener C, Neumaier M. Specific detection of carcinoembryonic antigenexpressing tumor cells in bone marrow aspirates by polymerase chain reaction. J Clin Oncol 1994;12:725-9.

11. Benevolo M, Mottolese M, Cosimelli M, Tedesco M, Giannarelli $\mathrm{D}$, Vasselli S, et al. Diagnostic and prognostic value of peritoneal immunocytology in gastric cancer. J Clin Oncol 1998;16:340611.
12. Yu W, Whang I, Suh I, Averbach A, Chang D, Sugarbaker PH. Prospective randomized trial of early postoperative intraperitoneal chemotherapy as an adjuvant to resectable gastric cancer. Ann Surg 1998;228:347-54.

13. Hagiwara A, Takahashi T, Kojima O, Sawai K, Yamaguchi T, Yamane $\mathrm{T}$, et al. Prophylaxis with carbon-absorbed mitomycin against peritoneal recurrence of gastric cancer. Lancet 1992;339: 629-31. 